\title{
Meta
}

Journal des traducteurs

Translators' Journal

\section{VAN HOOF, Henri (1993) : Dictionnaire universel des traducteurs, Genève, Éditions Slatkine, XIV + 414 p.}

\section{Paul A. Horguelin}

Volume 39, numéro 3, septembre 1994

URI : https://id.erudit.org/iderudit/003128ar

DOI : https://doi.org/10.7202/003128ar

Aller au sommaire du numéro

Éditeur(s)

Les Presses de l'Université de Montréal

ISSN

0026-0452 (imprimé)

1492-1421 (numérique)

Découvrir la revue

Citer ce compte rendu

Horguelin, P. A. (1994). Compte rendu de [VAN HOOF, Henri (1993) :

Dictionnaire universel des traducteurs, Genève, Éditions Slatkine, XIV + 414 p.]

Meta, 39(3), 475-477. https://doi.org/10.7202/003128ar

Ce document est protégé par la loi sur le droit d'auteur. L'utilisation des services d'Érudit (y compris la reproduction) est assujettie à sa politique d'utilisation que vous pouvez consulter en ligne.

https://apropos.erudit.org/fr/usagers/politique-dutilisation/
Cet article est diffusé et préservé par Érudit.

Érudit est un consortium interuniversitaire sans but lucratif composé de l’Université de Montréal, l’Université Laval et l’Université du Québec à Montréal. Il a pour mission la promotion et la valorisation de la recherche. https://www.erudit.org/fr/ 


\section{Comptes rendus}

VAN HOOF, Henri (1993): Dictionnaire universel des traducteurs, Genève, Éditions Slatkine, XIV + $414 \mathrm{p}$.

Traducteur et professeur de traduction, cofondateur de la Chambre belge des traducteurs et interprètes ainsi que de la revue Le Linguiste, depuis plus de trente ans Henri Van Hoof se signale régulièrement à notre attention par la publication d'ouvrages à un rythme que la retraite ne semble pas devoir ralentir. À côté de dictionnaires spécialisés et de manuels de traduction s'alignent deux bibliographies internationales et une Histoire de la traduction en Occident dont la publication, commencée en 1972 dans Le Linguiste, a connu son aboutissement en 1991 dans une édition revue et augmentée, synthèse la plus complète à ce jour. Production éclectique où se manifestent néanmoins une prédilection pour les travaux de longue haleine et un intérêt soutenu, sinon une passion, pour l'histoire de la traduction.

Le Dictionnaire universel des traducteurs se place donc sous le signe de la continuité, comme l'indique d'ailleurs l'auteur dans son Avant-propos, établissant une filiation entre sa Bibliographie internationale, son Histoire de la traduction et ce recensement des «acteurs mêmes de la traduction». Il s'agit d'une nouvelle contribution au projet d'histoire générale de la traduction, projet qui n'a guère progressé depuis sa conception au congrès de la FIT de 1966, mais auquel le Comité pour l'histoire de la traduction (que préside Jean Delisle et dont $\mathrm{H}$. Van Hoof est membre) vient de donner un regain de vie: une histoire thématique de la traduction doit paraître en 1996. (Rappelons que les lecteurs de Meta ont eu la primeur du Dictionnaire universel, dont les premières pages (lettres A et B) ont été publiées par tranches dans la revue, entre 1987 et 1991.)

La première question que soulève un dictionnaire biographique, tout comme une anthologie, est celle des critères de sélection : qui mettre ou omettre? Dans le cas présent, c'est «l'accès aux sources» qui a été déterminant : encyclopédies, histoires de la littérature, revues de traduction (la bibliographie des ouvrages consultés se trouve en pages liminaires) pour les «anciens»; associations professionnelles, maisons d'édition, palmarès de prix de traduction et «des centaines de traducteurs», pour la période contemporaine. Le résultat de cette quête est une liste de quelque 6500 noms, de A (AAFJES Bertus) à Z (ZWEIG Stefan), remontant dans le temps jusqu'aux premiers traducteurs latins (III ${ }^{\mathrm{e}}$-II ${ }^{\mathrm{e}}$ siècle av. J.-C.) et où sont représentés les cinq continents, mais de façon très inégale (part du lion pour l'Europe, une demi-douzaine d'élus pour l'Afrique) - au total plus de 60 pays, ce qui est somme toute une proportion raisonnable. C'est le critère de l'accès aux sources qui explique sans doute des absences remarquées (pays du Maghreb, Mexique) ou d'apparentes surreprésentations (Arménie, ex-Yougoslavie). Le Canada fait bonne figure avec 22 inscriptions, 26 si l'on compte le père Brébeuf, qui «a traduit le Catéchisme en "langage canadais"», deux traducteurs ecclésiastiques de passage... et «l'Américain David Homel», mentionné sous le nom de sa traductrice franco-belge.

L'Avant-propos nous avertit: «Dans leur impressionnante cohorte qui traverse les siècles défilent des saints et des hommes d'Église, des souverains et des princes, des 
hommes politiques et des chefs d'armée, des écrivains et des savants, des acteurs et des chansonniers, voire de "simples mortels" qui ont fait de la traduction un métier», ou tout au moins une activité secondaire. De fait, l'ordre alphabétique ménage de curieux voisinages, dans le temps et dans l'espace, et des rencontres inattendues: Catherine II, Maurice Chevalier, Élisabeth ${ }^{\mathrm{re}}$, Freud, Thomas Jefferson, Lénine, Louis XIII, Paul VI, Simone Signoret, Boris Vian... Exceptionnellement, quelques non-traducteurs se glissent dans la cohorte, notamment des théoriciens ou pédagogues (Catford, Darbelnet, Fedorov) et des déchiffreurs d'écriture (Champollion, Virolleaud, Young).

Passons maintenant en revue la présentation des articles, qui suit un schéma identique. Sur une première ligne, en caractères gras, figurent le patronyme, suivi du prénom et, entre parenthèses, des années de naissance et de décès. Viennent ensuite, numérotées: 1. une notice biographique; 2 . la liste des œuvres traduites et des publications originales traitant de traduction; 3 . le cas échéant, les distinctions obtenues. Notons d'abord que le prénom est parfois abrégé ou même omis, ce qui peut être gênant dans le cas des homonymes (p. ex., sept Leclerc sont répertoriés) et que très souvent les dates sont remplacées par la seule indication du siècle. Il est vrai que, dans bien des cas, le temps de recherche supplémentaire n'en valait pas la chandelle, mais dans d'autres (cf. Turgot), il suffisait d'ouvrir le Petit Larousse ou le Robert II. Les notices biographiques peuvent être très succinctes («Traducteur anglais») ou, au contraire, nous apprendre des choses intéressantes, voire révélatrices, sur la vie, la personnalité, l'activité professionnelle du traducteur. On constate ainsi que la traduction a été pour certains une activité familiale (cinq membres de la célèbre dynastie des Estienne, trois frères Thurot) ou conjugale (le couple Dacier est loin d'être unique). Traduire a aussi été un moyen de subsistance pour de nombreux exilés chassés par des troubles religieux ou politiques: révocation de l'édit de Nantes, révolution française ou bolchevique, régime nazi... D'autres notices viennent confirmer ce que nous savions déjà, grâce aux historiens de la traduction, sur le rôle des traducteurs dans les échanges interculturels et, d'une façon générale, dans le progrès de l'humanité.

La lecture du Dictionnaire universel est à l'occasion aussi divertissante qu'instructive. Peut-être ne saviez-vous pas qu'il y a eu des traducteurs précoces (Nicolas François était membre de quatre académies à l'âge de 13 ans), à vocation tardive (le duc de ClermontTonnerre entreprit à 67 ans la traduction des Euvres complètes d'Isocrate), polyglottes (Claude Hardy connaissait 36 langues anciennes et modernes), prolifiques (le jésuite allemand Albertinus remporte la palme avec 1600 titres!), consciencieux (Paul-Louis Courier «monte à la grecque sans étriers» avant d'entreprendre la traduction de deux traités d'équitation de Xénophon) ou téméraires (Dupré de Saint-Maur traduit sans connaître la langue de départ, et le soviétique Zochtchenko sans connaître la langue d'arrivée...).

$\grave{A}$ la rubrique des œuvres traduites, on trouve les titres, suivis du nom de l'auteur et de ses dates de naissance et de décès, ainsi que l'indication des langues de départ et d'arrivée, parfois la date de publication de la traduction. Les déclarations d'intention des traducteurs sont assez rares, de même que les jugements portés sur la qualité des traductions: qu'il s'agisse de louanges ou de critiques, $H$. Van Hoof semble se limiter aux cas qui font consensus. À l'occasion, toutefois, on lit qu'un Académicien français a commis en 1952 une version du Vieil homme et la mer «qui défigure complètement l'original». Shame on him ! La rubrique des distinctions recense presque exclusivement des prix de traduction décernés en Europe à des traducteurs et des traductrices littéraires.

Un ouvrage comme le Dictionnaire universel, où abondent les noms propres, les listes d'ouvrages dans plusieurs langues, les dates, sans parler de l'alternance des caractères italiques et romains, est un véritable «grenier à fautes», comme aurait dit Émile Littré, traducteur de Dante. C'est donc une agréable surprise de constater que le nombre des coquilles est tout à fait «dans les normes», tout comme celui des fautes vénielles 
(p. ex., vingt-et-un, Barthélémy, théorême, Villers-Cotterets, québecois, Chalons-sur-Saône et... Méta !). Aussi rares mais un peu plus dérangeantes que ces broutilles sont les erreurs concernant les titres d'ouvrage (p. ex., De natura rerum (et non De rerum natura) de Lucrèce, Les Remèdes d'amour (et non Le remède) d'Ovide, De la traduction ou règles pour apprendre à traduire (et non à bien traduire) de Gaspard de Tende), ou les éléments bio-bibliographiques (p. ex., Perrot d'Ablancourt est mort à son château d'Ablancourt et non à Paris, contrairement à ce qu'indique également le Robert $I I$, Marcel Proust a traduit La Bible d'Amiens et Sésame et les lys de Ruskin, et non Les Plaisirs et les jours, qui est une œuvre originale, et Jean-Paul Vinay n'a pas été «président de la Société des Traducteurs du Canada (STQ)» mais plus vraisemblablement de la Société des traducteurs et interprètes du Canada (STIC).

Une chose frappe : la disproportion entre les notices de certains contemporains (en particulier les lauréats européens de prix de traduction), qui ont droit à une demi-colonne, et la portion congrue réservée à d'autres comme Henri Meschonnic: «1. Traducteur français. 2. A traduit du russe: La Structure du texte artistique (1973) de Jouri Lotman, etc.» Cet «etc.» est un bel euphémisme! Plusieurs autres notices omettent des éléments importants (p. ex., saint Jérôme, Huet, Nabokov). Toujours au chapitre des péchés par omission, la liste des «grands oubliés» est courte. Pour le domaine français, nous sommes allé voir si George Sand ne se cachait pas derrière Aurore Dupin, eh bien non! On ne peut pourtant pas soupçonner H. Van Hoof d'être misogyne, car une des choses qui étonne dans sa recension des siècles antérieurs au nôtre est justement le nombre des traductrices - alors que les anthologies se limitent habituellement à quelques noms. Vérification faite, tous les grands traducteurs britanniques sont également présents; toutefois, si l'on estime que la préséance devrait être accordée à ceux qui ont laissé des réflexions sur la traduction, ajoutons au moins Sherburne pour le XVII (c'est lui qui a rétabli en Angleterre le vrai sens du Nec verbum verbo d'Horace) et Guthrie pour le XVIII (traducteur de Cicéron, il suit les débats de la Chambre des communes pour mieux rendre l'éloquence de l'orateur romain...). Dans le cas du Canada, enfin, il nous semble qu'à côté de Philippe Aubert de Gaspé, traducteur de Walter Scott, devrait figurer Pamphile Le May, auteur de la traduction française d'Évangéline, le célèbre poème de l'Américain Longfellow.

En relevant ces quelques erreurs ou lacunes, nous répondons à l'invitation de l'auteur dans son Avant-propos: «Nous souhaitons que cet inventaire ne soit qu'un point de départ et que le lecteur nous signale les omissions importantes, nous communique des précisions complémentaires, bref, participe activement à cet hommage au traducteur de tous les temps.» C'est ainsi effectivement que le Dictionnaire universel des traducteurs sera un ouvrage de référence sûr pour les chercheurs, historiens et, d'une façon générale, les traducteurs et traductrices qui s'intéressent au passé, lointain ou récent, de leur profession. Pour notre part, nous formulerons le souhait que dans une prochaine édition on puisse lire à l'article VAN HOOF Henri (1923- ): «3. Prix de la FIT décerné pour sa contribution à l'histoire de la traduction.»

En attendant, le bénédictin de la traduction a repris sa plume. Pas moins de trois ouvrages sont sur le métier: deux dictionnaires spécialisés et une Petite histoire de la traduction médicale... Retraite oisive, connaît pas !

Paul A. Horguelin 